\title{
Eficiência na produção de frutos e alocação relativa de nutrientes em cultivares de cafeeiro ${ }^{1}$
}

\author{
José Francisco Teixeira do Amaral ${ }^{2}$, Herminia Emilia Prieto Martinez ${ }^{3}$, Bruno Galveas Laviola ${ }^{4}$, \\ Elpidio Inácio Fernandes Filho ${ }^{5}$, Cosme Damião Cruz ${ }^{6}$
}

\section{RESUMO}

Considerando a baixa produtividade das plantas em solos de menor fertilidade natural e o alto custo dos insumos agrícolas, torna-se necessária a seleção de cultivares mais eficientes na absorção e utilização dos nutrientes minerais. Foram avaliados quatro cultivares de cafeeiro arábica (Acaiá IAC 474 19, Icatu Amarelo IAC 3282, Rubi MG 1192 e Catuaí Vermelho IAC 99) quanto à eficiência na produção de frutos e alocação relativa de nutrientes. O experimento foi conduzido em Viçosa - MG, em condições de campo, no delineamento experimental em blocos ao acaso, envolvendo quatro cultivares, quatro repetições e três níveis de adubação (baixo, normal e alto). As parcelas úteis constituíram-se de nove plantas espaçadas de 2 x 1 m. O cultivar Icatu Amarelo IAC 3282 foi o mais produtivo no ambiente com restrição de nutrientes, enquanto Rubi MG 1192 e Catuaí Vermelho IAC 99 mostraram-se mais produtivos em ambientes com alto suprimento de nutrientes. A eficiência de produção de café em coco por unidade de P, Ca, Mg e B acumulados na planta foi maior no nível alto de adubação. Os cultivares Rubi MG-1192 e Catuaí Vermelho IAC 99 apresentaram maior eficiência de utilização de nutrientes para produção de frutos no nível alto de adubação. Considerando a média de alocação relativa de nutrientes nos frutos para os quatro cultivares, no nível normal de adubação, verificou-se que eles possuem 38,1\% do N, 46,34\% do P, 40,19\% do S, 42,68\% do K, 13,19\% do Ca, 25,04\% do Mg, 40,63\% do Cu, 19,49\% do Zn e $17,73 \%$ do $B$.

Palavras - Chave: Coffea arabica L., nutrição mineral, eficiência nutricional.

\section{ABSTRACT}

\section{Bean production efficiency and relative allocation of nutrients of four coffee varieties}

Considering the low productivity of plants in soil of lower natural fertility and the high cost of fertilizers, the selection of cultivars more efficient in the absorption and use of mineral nutrients is a necessity. Four coffee varieties were evaluated for mineral nutrient use efficiency in the production of beans, as well as the relative allocation of these nutrients in reproductive organs. The experiment was carried-out in field conditions in an experimental area of the Universidade Federal de Viçosa, Viçosa, Minas Gerais State, Brazil. The treatments were distributed in randomized blocks design, in a factorial arrangement (4 x 3) x 4, consisting of four coffee varieties (Acaiá IAC-474-19, Icatu Amarelo IAC-3282, Rubi MG1192 and Catuaí Vermelho IAC-99), three fertilization levels (lower, adequate and high), with four replications. The plot useful area consisted of nine plants with spacing of 2 x $1 \mathrm{~m}$. The variety Icatu Amarelo IAC-3282 was the most productive in the condition of low nutrient input, while Rubi MG-1192 and Catuaí Vermelho IAC-99 were more productive with high

Recebido para publicação em julho de 2008 e aprovado em fevereiro de 2010

${ }^{1}$ Parte da tese de Doutorado do primeiro autor apresentada à Universidade Federal de Viçosa - UFV. Trabalho financiado pelo CNP\&D-Café.

${ }^{2}$ Engenheiro-Agrônomo, Doutor, Departamento de Engenharia Rural do Centro de Ciências Agrárias da Universidade Federal do Espírito Santo/UFES, Alto Universitário, s/n, Cx. P. 16, 29500-000, Alegre, ES, Brasil. jfamaral@cca.ufes.br.

${ }^{3}$ Engenheira-Agrônoma, Doutora. Departamento de Fitotecnia da Universidade Federal de Viçosa/UFV, Av. Peter Henry Rolfs, s/n, 36570-000, Viçosa, MG, Brasil. herminia@ufv.br. ${ }^{4}$ Engenheiro-Agrônomo, Doutor, Embrapa Agroenergia, Parque Estação Biológica - PqEB s/n, CEP 70770-901, Brasïlia, D , Brasil. bruno.laviola@embrapa.br.

${ }^{5}$ Engenheiro- Agrônomo, Doutor. Departamento de Solos da Universidade Federal de Viçosa/UFV, Av. Peter Henry Rolfs, s/n, 36570-000, Viçosa, MG, Brasil. elpidio@ufv.br.

${ }^{6}$ Engenheiro-Agrônomo, Doutor. Departamento de Biologia Geral da Universidade Federal de Viçosa/UFV, Av. Peter Henry Rolfs, s/n, 36570-000, Viçosa, MG, Brasil. cdcruz@ufv.br. 
nutrient input. The production of dry coffee per unit of P, Ca, Mg and B accumulated in the plant was larger at the high fertilization level. The varieties Rubi MG-1192 and Catuaí Vermelho IAC-99 showed greater efficiency of nutrient use for production of beans at the high level of fertilization. Considering the mean of relative allocation of nutrients in the beans, of the four varieties, at the usual level of fertilization, it was found that they have $38.1 \%$ of N, $46.3 \%$ of P, $40.2 \%$ of S, $42.7 \%$ of K, $13.2 \%$ of the Ca, $25.0 \%$ of $\mathrm{Mg}, 40.6 \%$ of the $\mathrm{Cu}, 19.5 \%$ of $\mathrm{Zn}$ and $17.7 \%$ of B.

Key words: Coffea arabica L., mineral nutrition, nutritional efficiency.

\section{INTRODUÇÃO}

A seleção de material genético que melhor se adapte à condição de fertilidade do solo mais baixa tem sido uma preocupação constante, sendo desejáveis as espécies com maior capacidade de absorver e utilizar os nutrientes (Morais et al., 1990). Do ponto de vista nutricional, uma espécie/genótipo superior é aquela capaz de se desenvolver e ter boa produção em condições desfavoráveis de fertilidade do solo, tendo habilidade em absorver os nutrientes necessários, em menor quantidade, e, ou, distribuí-los de maneira mais eficiente nos diversos componentes da planta, sem comprometer a produtividade (Furlani et al., 1984).

Com base na capacidade de produzir matéria seca por unidade de nutriente, as plantas podem ser chamadas nutricionalmente de "eficientes" ou "ineficientes" (Vose, 1987). A eficiência nutricional em plantas pode estar relacionada à eficiência de absorção, translocação e utilização de nutrientes. A eficiência de absorção está relacionada à taxa de absorção de nutrientes por unidade de comprimento ou de massa de raiz e pode ser avaliada em estudos de cinética de absorção de nutrientes (Baligar e Fageria, 1998).

Devido ao fato de a cada ano serem lançadas no mercado novas variedades de cafeeiros com maior capacidade produtiva, e das lavouras cafeeiras se expandirem para novas fronteiras agrícolas, com solos de fertilidade variável, torna-se necessário compreender melhor as eficiências de absorção, translocação e redistribuição dos nutrientes nos diferentes cultivares de café (Laviola et al., 2007). Embora existam muitas pesquisas com nutrição mineral em cafeeiro, pouco se sabe sobre as diferenças entre cultivares no uso e na alocação de nutrientes.

O objetivo deste trabalho foi estudar a eficiência dos cultivares de cafeeiro na produção de frutos e sua alocação relativa de nutrientes em diferentes órgãos.

\section{MATERIAL E MÉTODOS}

O experimento foi conduzido em uma Área Experimental do Departamento de Fitotecnia, pertencente à Univer- sidade Federal de Viçosa, sendo instalado em outubro de 1998. Foram utilizados quatro cultivares de cafeeiro arábica: Acaiá IAC 474 19, Icatu Amarelo IAC 3282, Rubi MG 1192 e Catuaí Vermelho IAC 99.

O solo onde foi conduzido o experimento é classificado como Latossolo Vermelho-Amarelo Distrófico, e suas características químicas são apresentadas na Tabela 1.

O experimento constituiu-se de três níveis de adubação e correção da acidez do solo: baixo, normal (recomendado para a cultura) e alto. O delineamento experimental empregado foi em blocos completos casualizados, e os tratamentos foram distribuídos num arranjo fatorial $4 \times 3$, sendo quatro cultivares e três níveis de adubação, com quatro repetições, totalizando 48 unidades experimentais. Cada parcela foi constituída de 25 plantas, espaçadas de $2 \times 1 \mathrm{~m}$, dispostas em cinco fileiras, ocupando uma área de $50 \mathrm{~m}^{2}$. Considerou-se como parcela útil as nove plantas dispostas no centro das três fileiras centrais da parcela.

As plantas que receberam o nível normal de adubação N, P e K tiveram como base a marcha de acúmulo, considerando-se a média de nutrientes acumulados para os cultivares Mundo Novo e Catuaí, conforme Malavolta (1993). O Ca e Mg foram fornecidos via calcário dolomítico, segundo a análise de solo, considerando-se $60 \%$ de saturação por bases como ideal para o cafeeiro (Guimarães et al., 1999). O enxofre foi fornecido como elemento acompanhante de fertilizantes nitrogenados e fosfatados.

A aplicação de corretivos e fertilizantes para o nível normal constou do seguinte: a) plantio: $20 \mathrm{~g} /$ cova de calcário dolomítico e $48 \mathrm{~g} /$ cova de $\mathrm{P}_{2} \mathrm{O}_{5}$; b) pós-plantio: $8 \mathrm{~g} /$ cova de $\mathrm{N}$ em três aplicações e $20 \mathrm{~g} /$ cova de $\mathrm{K}_{2} \mathrm{O}$ em duas aplicações, em intervalos de 30 dias; c) correção da acidez do solo: foi realizada anualmente, mediante aplicação manual de calcário dolomítico na faixa de plantio, considerando-se uma profundidade de incorporação de $5 \mathrm{~cm}$; d) adubação de primeiro, segundo e terceiro anos: foram realizadas via fertirrigação, considerando-se uma eficiência de recuperação de $90 \%$ para o N, $80 \%$ para o K e $70 \%$ para o P. Os nutrientes $\mathrm{N}$, P e K foram aplicados no solo localizadamente pela utilização de fertirrigação por gotejamento, com o su-

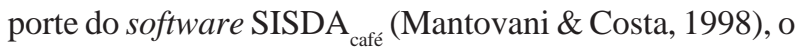


Tabela 1 - Características químicas do solo da área experimental

\begin{tabular}{|c|c|c|c|c|c|c|c|c|c|c|c|c|}
\hline $\begin{array}{l}\mathrm{pH} \\
\text { em } \mathrm{H}_{2} \mathrm{O}\end{array}$ & $\mathbf{P}$ & $\mathbf{K}$ & $\mathrm{Na}^{+}$ & $\mathrm{Ca}^{2+}$ & $\mathbf{M g}^{2+}$ & $\mathrm{Al}^{3+}$ & SB & $\mathbf{H}+\mathbf{A l}$ & $\begin{array}{c}\text { CTC } \\
\text { efetiva }\end{array}$ & $\begin{array}{l}\text { CTC } \\
\text { total }\end{array}$ & V & m \\
\hline & - & $18 / 4$ & 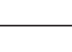 & \multicolumn{7}{|c|}{$\mathrm{cmol}_{\mathrm{c}} / \mathrm{dm}^{3}$} & 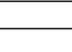 & \\
\hline 5,6 & 17,6 & 92 & 8 & 1,94 & 0,72 & 0 & 2,94 & 2,69 & 2,94 & 5,63 & 52,2 & 0 \\
\hline
\end{tabular}

$\mathrm{SB}=$ Soma de bases;

$\mathrm{V}(\%)=$ porcentagem de saturação por bases;

$\mathrm{m}(\%)=$ porcentagem de saturação por alumínio;

$\mathrm{Al}^{3+}, \mathrm{Ca}^{2+}$ e $\mathrm{Mg}^{2+}$ extraídos com KCl 1 mol L-1; P e K extraídos com extrator biácido ( $\mathrm{HCl}^{-1} \mathrm{~mol} \mathrm{~L}^{-1}$ e $\mathrm{H}_{2} \mathrm{SO}_{4} 25 \mathrm{~mol} \mathrm{~L}^{-1}$ ); pH em $\mathrm{H}_{2} \mathrm{O}$ relação $1: 2,5$.

qual também define a lâmina a ser aplicada segundo informações meteorológicas diárias fornecidas pelo Departamento de Engenharia Agrícola da Universidade Federal de Viçosa. O N foi fornecido pela aplicação alternada de sulfato de amônio, ureia e nitrato de amônio; o P pela aplicação de MAP (fosfato monoamônio); e o K foi adicionado por meio do cloreto de potássio. Um quarto da exigência anual de nutrientes calculada foi aplicado no período de abril a agosto, e os 3/4 restantes de setembro a março e; e) micronutrientes: Zn, B e Cu foram supridos por meio de uma aplicação foliar anual, utilizando-se sulfato de zinco, ácido bórico e oxicloreto de cobre na concentração de 4 g/L para o nível normal.

Nos níveis baixo e alto, as plantas receberam, respectivamente, 0,4 e 1,4 vezes a recomendação feita para o nível normal.

As avaliações foram iniciadas no mês de maio de 2001, coincidindo com a colheita dos frutos. Para a avaliação da produtividade foram consideradas as nove plantas da parcela útil, enquanto para o estudo das eficiências nutricionais foi cortada uma planta em cada parcela experimental, cuja parte aérea foi fracionada em caule, ramos, folhas e frutos. A contribuição das raízes foi estimada com base em amostragens.

As amostras da parte aérea foram identificadas, lavadas com água deionizada, acondicionadas em sacos de papel e secas em estufa com circulação forçada de ar, a 70 ${ }^{\circ} \mathrm{C}$, até massa constante, conforme descrito por Jones Junior et al. (1991). A seguir, amostras do material vegetal seco foram moídas em moinho tipo Wiley, com peneira de 0,841 mm para a efetivação das análises minerais.

Para estimar o comprimento e o peso de matéria seca de raízes, foram retiradas com trado cilíndrico de 4,5 cm de diâmetro 12 amostras de uma planta em cada parcela. Essas amostras foram tomadas à 15, 30 e $45 \mathrm{~cm}$ do tronco, no sentido dos quatro pontos cardeais, até $40 \mathrm{~cm}$ de profundidade. As amostras foram lavadas para separar as raízes do solo, identificadas e dispostas em lâmina de vidro para processamento das imagens em um microcomputador ligado a um scanner HP Scanjet 4C. As imagens obtidas foram processadas pelo software QUANTROOT, elaborado pelo Departamento de Solos da Universidade Federal de Viçosa, especialmente desenvolvido para determinação do comprimento de raízes. Depois de determinado o comprimento de raízes, os resultados foram extrapolados para o volume de solo ocupado pelas raízes da planta $\left(1,25664 \mathrm{~m}^{3}\right)$, ou seja, o volume de um cilindro com raio de $1 \mathrm{~m}$ e altura de $0,40 \mathrm{~m}$.

Após o processamento das imagens, as amostras foram secas em estufa de circulação forçada de ar a $70{ }^{\circ} \mathrm{C}$, até peso constante, para determinação do peso de matéria seca de raízes. Esse peso foi também extrapolado para o volume de solo ocupado pelo sistema radicular da planta. Foram, então, separadas amostras desse material para determinação dos teores e conteúdos dos nutrientes.

Foram determinados os teores dos macro e dos micronutrientes $\mathrm{Cu}$, Zn e B nos órgãos da planta. Para determinação dos teores de N, P, K, Ca, Mg, S, Cu e Zn, o material vegetal, seco e moído, foi submetido à digestão sulfúrica (Jackson, 1958) e à nitroperclórica (Johnson e Ulrich, 1959), bem como à extração com água, em banhomaria a $45^{\circ} \mathrm{C}$, durante $1 \mathrm{~h}$, para análise do nitrato.

As amostras oriundas da digestão sulfúrica foram utilizadas para as determinações dos teores de $\mathrm{N}$ amoniacal, enquanto que as processadas pela digestão nitroperclórica foram usadas para as determinações dos teores de P, K, Ca, Mg, S, Cu e Zn.

$\mathrm{O} \mathrm{N}$ amoniacal, o nitrato e o fósforo foram determinados por colorimetria, segundo os métodos descritos por Cataldo et al. (1975), Jackson (1958) e Braga e Defelipo (1974). O K foi dosado por fotometria de chama, enquanto o $\mathrm{Ca}, \mathrm{Mg}$, Cu e Zn foram quantificados por espectrofotometria de absorção atômica e o S por turbidimetria do sulfato (Jackson, 1958). O teor de B foi obtido por colorimetria pelo método da Azometina-H, após digestão das amostras por via seca (calcinação em mufla a $550{ }^{\circ} \mathrm{C}$ Bingham, 1982).

O conteúdo de macro e micronutrientes foi obtido pela multiplicação do teor do nutriente (g/kg e mg/kg) pela massa da matéria seca (em g) em cada parte da planta, obtendo-se os resultados respectivamente em mg e $\mu \mathrm{g}$ por planta.

Os índices de eficiência nutricional foram obtidos a partir de relações entre produção de matéria seca e con- 
teúdos de nutrientes em cada parte da planta, conforme as relações apresentadas a seguir:

Eficiência de utilização de nutrientes para produção de frutos (EUFR):

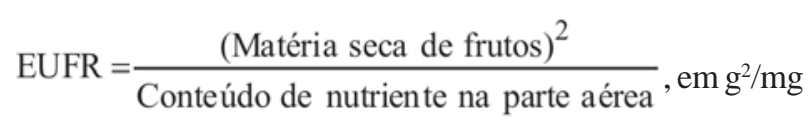

e $g^{2} / \mu g$ para macro e micronutrientes, respectivamente.

Alocação relativa de nutrientes no caule (ANC):

$\mathrm{ANC}=\frac{\text { Conteúdo de nutriente no caule }}{\text { Conteúdo de nutriente na parte aérea }} \times 100$, em $\%$ para macro e micronutrientes.

Alocação relativa de nutrientes nos ramos (ANR):

$\mathrm{ANR}=\frac{\text { Conteúdo de nutriente nos ramos }}{\text { Conteúdo de nutriente na parte aérea }} \times 100$, em \% para macro e micronutrientes.

Alocação relativa de nutrientes nas folhas (ANF):

$\mathrm{ANF}=\frac{\text { Conteúdo de nutriente nas folhas }}{\text { Conteúdo de nutriente na parte aérea }} \times 100$, em \% para macro e micronutrientes.

Alocação relativa de nutrientes nos frutos (ANFR):

ANFR $=\frac{\text { Conteúdo de nutriente no fruto }}{\text { Conteúdo de nutriente na parte aérea }} \times 100$, em $\%$ para macro e micronutrientes.

\section{RESULTADOS E DISCUSSÃO}

Considerando a média de produtividade (Tabela 2), observa-se que essa foi maior no nível alto de adubação. Os cultivares Rubi MG 1192 e Catuaí Vermelho IAC 99 apresentaram produtividade mais elevada nos níveis de adubação normal e alto, enquanto o Acaiá IAC 47419 foi o que teve a menor produtividade nos três ambientes. O cultivar Icatu Amarelo IAC 3282 foi o mais produtivo no nível baixo de adubação, tendo sua produtividade decrescido com o aumento das doses de nutrientes no solo. Pode-se sugerir que o Icatu Amarelo IAC 3282 seja um cultivar com menor exigência nutricional, apresentando, ainda, maior eficiência de utilização de nutrientes em condições de baixo nível de adubação, em comparação com os demais.

Ensaio regional de linhagens comerciais de cultivares de cafeeiros arábica instalado em Belizário, distrito de Muriaé-MG, evidenciou produtividade de 23,73; 23,73; e 23,47 sacas/ha de café beneficiado para os cultivares Icatu Amarelo IAC-3282, Catuaí Vermelho IAC-99 e Rubi MG1192, respectivamente (Moura et al., 2000). Comparando esses resultados com os obtidos neste trabalho, há uma indicação de que esses três cultivares comportaram-se melhor em Viçosa, onde suas produtividades foram 23,9; 38,8; e 40,1 sacas/ha de café beneficiado, respectivamente, embora o espaçamento utilizado nos dois locais fosse diferente.

No nível mais baixo de adubação, que corresponde a uma redução de $60 \%$ no fornecimento de adubos em relação ao recomendado para a cultura (normal), os cultivares Icatu Amarelo IAC-3282 e Acaiá IAC-474-19 produziram, respectivamente, 30,2 e 12,4 sacas/ha de café beneficiado. Entretanto, no nível normal de adubação os cultivares Rubi MG-1192 e Catuaí Vermelho IAC-99 tiveram as melhores produções, sem diferir do cultivar Icatu Amarelo IAC-3282, porém superiores ao Acaiá IAC-474-19. Aplicando-se 1,4 vezes a dose de adubo recomendada (nível alto), os cultivares Rubi MG-1192 e Catuaí Vermelho IAC99 destacaram-se, diferindo estatisticamente dos cultivares Acaiá IAC-474-19 e Icatu Amarelo IAC-3282. Dessa forma, pode-se dizer que os cultivares Rubi MG-1192 e Catuaí Vermelho IAC-99, além de produtivos, apresentaram boas respostas à adubação. Esses cultivares aumentaram a produção em 60 e 51\% do nível baixo para o normal e em 21 e $29 \%$ desse para o alto, respectivamente. O Acaiá IAC-474-19 foi menos produtivo e alcançou incrementos na produção de 47,5\% do nível baixo para o normal e de $41 \%$ desse para o alto. A produtividade do Icatu Amarelo IAC-3282 teve redução de aproximadamente 20\% do nível baixo para o normal, e desse para o nível alto praticamente não variou. Nesse contexto, pode-se inferir

Tabela 2 - Produtividade de quatro cultivares de café arábica, com três anos de idade, submetidos a três níveis de adubação

\begin{tabular}{lcccc}
\hline \multirow{2}{*}{ Cultivar } & \multicolumn{3}{c}{ Nível de adubação } & \multirow{2}{*}{ Média } \\
\cline { 2 - 3 } & Baixo & Normal & Alto & \\
\cline { 2 - 3 } & & sacas/ha de café beneficiado & $18,8^{\mathrm{B}}$ \\
Acaiá IAC-474-19 & $12,4^{\mathrm{B}}$ & $18,3^{\mathrm{B}}$ & $25,8^{\mathrm{B}}$ & $26,5^{\mathrm{A}}$ \\
Icatu Amarelo IAC-3282 & $30,2^{\mathrm{A}}$ & $25,2^{\mathrm{AB}}$ & $23,9^{\mathrm{B}}$ & $31,3^{\mathrm{A}}$ \\
Rubi MG-1192 & $20,7^{\mathrm{AB}}$ & $33,2^{\mathrm{A}}$ & $40,1^{\mathrm{A}}$ & $29,6^{\mathrm{A}}$ \\
Catuaí Vermelho IAC-99 & $19,9^{\mathrm{AB}}$ & $30,1^{\mathrm{A}}$ & $38,8^{\mathrm{A}}$ & $32,2^{\mathrm{a}}$ \\
Média & $20,8^{\mathrm{c}}$ & $26,7^{\mathrm{b}}$ & \\
\hline
\end{tabular}

Médias seguidas pela mesma letra maiúscula na coluna e minúscula na linha não diferem estatisticamente entre si a $10 \%$, pelo teste de Tukey.

Rev. Ceres, Viçosa, v. 57, n.2, p. 253-262, mar/abr, 2010 
que o cultivar Icatu Amarelo IAC-3282 destacou-se, sendo mais produtivo que os demais, quando submetido a baixos níveis de adubação. Os cultivares Rubi MG-1192 e Catuaí Vermelho IAC-99 foram os mais produtivos nos níveis normal e alto de adubação e responderam ao incre- mento de adubação, enquanto o cultivar Acaiá IAC-47419, embora responda bem à adubação, apresentou menores produções quando comparados com os demais.

Quanto à eficiência de utilização de nutrientes para produção de frutos (Tabela 3) percebem-se diferenças

Tabela 3 - Eficiência de utilização de nutrientes para produção de frutos ${ }^{1}$ de quatro cultivares de café arábica, submetidos a três níveis de adubação

\begin{tabular}{|c|c|c|c|c|c|c|}
\hline \multirow{2}{*}{ Nutriente } & \multirow{2}{*}{ Cultivar } & \multicolumn{3}{|c|}{ Nível de Adubação } & \multirow{2}{*}{ Média } & \multirow{2}{*}{ CV (\%) } \\
\hline & & Baixo & Normal & Alto & & \\
\hline & Acaiá IAC-474-19 & $13,7325^{\mathrm{A}}$ & $7,9625^{\text {в }}$ & $13,2375^{\mathrm{BC}}$ & $11,6442^{\mathrm{A}}$ & \\
\hline \multirow[t]{5}{*}{$\mathrm{N}$} & Icatu Amarelo IAC-3282 & $20,2275^{A}$ & 18,0875 АВ & $10,5000^{\mathrm{C}}$ & $16,2717^{\mathrm{A}}$ & 47,34 \\
\hline & Rubi MG-1192 & $13,5975^{\mathrm{A}}$ & $22,7400^{\mathrm{A}}$ & $25,2250^{\mathrm{AB}}$ & $20,5208^{A}$ & \\
\hline & Catuaí Vermelho IAC-99 & $19,3850^{\mathrm{A}}$ & 18,2400 АВ & $27,8750^{\mathrm{A}}$ & $21,8333^{\mathrm{A}}$ & \\
\hline & Média & $16,7356^{\mathrm{a}}$ & $16,7575^{\mathrm{a}}$ & $19,2094^{\mathrm{a}}$ & & \\
\hline & Acaiá IAC-474-19 & $148,8525^{\mathrm{A}}$ & $128,4225^{\text {в }}$ & $221,1725^{\text {в }}$ & $166,1492^{\mathrm{A}}$ & \\
\hline \multirow[t]{5}{*}{$\mathrm{P}$} & Icatu Amarelo IAC-3282 & $260,6900^{\mathrm{A}}$ & 300,2475 АВ & $193,3450^{\text {в }}$ & $251,4275^{\mathrm{A}}$ & 44,63 \\
\hline & Rubi MG-1192 & $148,1950^{\mathrm{A}}$ & $375,0075^{\text {A }}$ & $491,0450^{\mathrm{A}}$ & $338,0825^{\text {A }}$ & \\
\hline & Catuaí Vermelho IAC-99 & $215,1100^{\mathrm{A}}$ & 296,2875 АВ & $505,2450^{\mathrm{A}}$ & $338,8808^{\mathrm{A}}$ & \\
\hline & Média & $193,2119^{b}$ & 274,9913 ab & $352,7019^{a}$ & & \\
\hline & Acaiá IAC-474-19 & $179,6275^{\text {A }}$ & $117,6600^{\text {в }}$ & $181,9925^{\text {в }}$ & $159,7600^{\mathrm{A}}$ & \\
\hline \multirow[t]{5}{*}{ S } & Icatu Amarelo IAC-3282 & $269,3800^{\mathrm{A}}$ & 252,6525 АВ & $144,4250^{\text {в }}$ & $222,1525^{\mathrm{A}}$ & 46,84 \\
\hline & Rubi MG-1192 & $169,1250^{\mathrm{A}}$ & $318,1300^{\mathrm{A}}$ & 330,4000 АВ & $272,5517^{\mathrm{A}}$ & \\
\hline & Catuaí Vermelho IAC-99 & $239,0650^{\mathrm{A}}$ & 256,6325 Ав & $385,4775^{\text {A }}$ & $293,7250^{\mathrm{A}}$ & \\
\hline & Média & $214,2994^{\text {a }}$ & $236,2688^{a}$ & $260,5738^{a}$ & & \\
\hline & Acaiá IAC-474-19 & $27,5150^{\mathrm{A}}$ & $18,5875^{\text {в }}$ & $28,6950^{\text {в }}$ & $24,9325^{\mathrm{A}}$ & \\
\hline \multirow[t]{5}{*}{ K } & Icatu Amarelo IAC-3282 & $42,0450^{\mathrm{A}}$ & 39,5650 АВ & $22,5750^{\text {в }}$ & $34,7283^{A}$ & 45,08 \\
\hline & Rubi MG-1192 & $27,2800^{\mathrm{A}}$ & $53,5725^{A}$ & $58,7100^{\mathrm{A}}$ & $46,5208^{A}$ & \\
\hline & Catuaí Vermelho IAC-99 & $38,5625^{\mathrm{A}}$ & 39,8100 АВ & $59,4275^{\mathrm{A}}$ & $45,9333^{\mathrm{A}}$ & \\
\hline & Média & $33,8506^{a}$ & $37,8838^{a}$ & $42,3519^{a}$ & & \\
\hline & Acaiá IAC-474-19 & $30,2625^{A}$ & $22,2750^{\text {в }}$ & 44,6075 вС & $32,3817^{\mathrm{A}}$ & \\
\hline \multirow[t]{5}{*}{$\mathrm{Ca}$} & Icatu Amarelo IAC-3282 & $45,2050^{\mathrm{A}}$ & $49,0750^{\mathrm{AB}}$ & $36,8925^{\mathrm{c}}$ & $43,7242^{\mathrm{A}}$ & 46,38 \\
\hline & Rubi MG-1192 & $32,1375^{\mathrm{A}}$ & $64,3325^{\mathrm{A}}$ & 79,5975 Ав & $58,6892^{\mathrm{A}}$ & \\
\hline & Catuaí Vermelho IAC-99 & $45,5675^{\mathrm{A}}$ & $44,9725 \mathrm{AB}$ & $95,5175^{\mathrm{A}}$ & $62,0192^{\mathrm{A}}$ & \\
\hline & Média & $38,2931^{b}$ & $45,1638^{\mathrm{b}}$ & $64,1538^{a}$ & & \\
\hline & Acaiá IAC-474-19 & $96,5525^{\mathrm{A}}$ & $64,2225^{\text {B }}$ & $111,4275^{\mathrm{BC}}$ & $90,7342^{\mathrm{A}}$ & \\
\hline \multirow[t]{5}{*}{$\mathrm{Mg}$} & Icatu Amarelo IAC-3282 & $138,4350^{\mathrm{A}}$ & $140,4800 \mathrm{AB}$ & $92,3000^{\mathrm{c}}$ & $123,7383^{\mathrm{A}}$ & 47,65 \\
\hline & Rubi MG-1192 & $92,1275^{\mathrm{A}}$ & $184,5675^{\mathrm{A}}$ & 221,5200 АВ & $166,0717^{\mathrm{A}}$ & \\
\hline & Catuaí Vermelho IAC-99 & $140,6825^{\text {A }}$ & $133,2725^{\mathrm{AB}}$ & $259,0475^{\text {A }}$ & $177,6675^{\mathrm{A}}$ & \\
\hline & Média & $116,9494^{\mathrm{a}}$ & $130,6356^{\text {a }}$ & $171,0738^{a}$ & & \\
\hline & Acaiá IAC-474-19 & $11,5825^{\mathrm{A}}$ & $10,7650^{\mathrm{A}}$ & 13,0075 Ав & $11,7850^{\mathrm{A}}$ & \\
\hline \multirow[t]{5}{*}{$\mathrm{Cu}$} & Icatu Amarelo IAC-3282 & $17,3375^{\mathrm{A}}$ & $21,5800^{\mathrm{A}}$ & $10,3475^{\text {в }}$ & $16,4217^{\mathrm{A}}$ & 48,48 \\
\hline & Rubi MG-1192 & $11,1600^{\mathrm{A}}$ & $25,1275^{\text {A }}$ & $24,8700^{\text {A }}$ & $20,3858^{\mathrm{A}}$ & \\
\hline & Catuaí Vermelho IAC-99 & $16,5100^{\mathrm{A}}$ & $20,3025^{\mathrm{A}}$ & 24,4375 АВ & $20,4167^{\mathrm{A}}$ & \\
\hline & Média & $14,1475^{\mathrm{a}}$ & $19,4438^{\mathrm{a}}$ & $18,1656^{\text {a }}$ & & \\
\hline & Acaiá IAC-474-19 & $23,3650^{\mathrm{A}}$ & $13,0275^{\text {в }}$ & 22,7325 Ав & $19,7083^{\mathrm{A}}$ & \\
\hline \multirow[t]{5}{*}{$\mathrm{Zn}$} & Icatu Amarelo IAC-3282 & $34,4200^{\mathrm{A}}$ & 31,9825 АВ & $17,3300^{\text {в }}$ & $27,9108^{\mathrm{A}}$ & 52,53 \\
\hline & Rubi MG-1192 & $23,3475^{\mathrm{A}}$ & $43,9500^{\mathrm{A}}$ & 44,1500 АВ & $37,1492^{\mathrm{A}}$ & \\
\hline & Catuaí Vermelho IAC-99 & $29,7225^{A}$ & $32,0575 \mathrm{AB}$ & $49,6375^{\mathrm{A}}$ & $37,1392^{\mathrm{A}}$ & \\
\hline & Média & $27,7138^{a}$ & $30,2544^{\mathrm{a}}$ & $33,4625^{a}$ & & \\
\hline & Acaiá IAC-474-19 & $7,9550^{\mathrm{A}}$ & $9,3800^{\text {в }}$ & $12,4200^{\text {в }}$ & $9,9183^{\mathrm{A}}$ & \\
\hline \multirow[t]{4}{*}{ B } & Icatu Amarelo IAC-3282 & $12,8625^{\mathrm{A}}$ & $24,1350^{\mathrm{A}}$ & $12,1650^{\mathrm{B}}$ & $16,3875^{\mathrm{A}}$ & 46,18 \\
\hline & Rubi MG-1192 & $7,9800^{\mathrm{A}}$ & $25,1700^{\mathrm{A}}$ & $28,8850^{\mathrm{A}}$ & $20,6783^{A}$ & \\
\hline & Catuaí Vermelho IAC-99 & $11,8175^{\mathrm{A}}$ & 18,5850 АВ & $31,6100^{\mathrm{A}}$ & $20,6708^{A}$ & \\
\hline & Média & $10,1538^{\mathrm{b}}$ & $19,3175^{\text {a }}$ & $21,2700^{a}$ & & \\
\hline
\end{tabular}

Médias seguidas pela mesma letra maiúscula na coluna e minúscula na linha não diferem estatisticamente entre si a $10 \%$, pelo teste de Tukey.

${ }^{1 /}$ EUFR $=\frac{(\text { Matéria seca de frutos })^{2}}{\text { Conteúdo de nutriente na parte aérea }}, \mathrm{em} \mathrm{g}^{2} / \mathrm{mg} \mathrm{e} \mathrm{g}^{2} / \mathrm{mg}$ para macro e micronutrientes, respectivamente. 
entre os cultivares somente nos níveis de adubação normal e alto que apresentam o mesmo padrão para todos os nutrientes. Observa-se tendência de superioridade do Rubi MG 1192 e do Catuaí Vermelho IAC 99, principalmente no nível mais alto de adubação. Essa melhor utilização de nutrientes para produção de frutos pode explicar o bom desempenho desses cultivares quanto à produtividade nesses dois níveis de adubação.

Não houve diferenças entre os cultivares na alocação relativa de nutrientes no caule (Tabela 4). A alocação de $\mathrm{N}, \mathrm{P}, \mathrm{K}$ e Mg no caule foi maior no nível baixo de adubação em comparação aos demais níveis.

Tabela 4 - Alocação relativa de nutrientes no caule² de quatro cultivares de café arábica, submetidos a três níveis de adubação

\begin{tabular}{|c|c|c|c|c|c|c|}
\hline Nutriente & Cultivar & $\frac{\text { Nível de Adubação }}{\text { Baixo }}$ & $\begin{array}{c}\text { Média } \\
\text { Normal }\end{array}$ & $\begin{array}{c}\text { CV (\%) } \\
\text { Alto }\end{array}$ & Média & CV (\%) \\
\hline & Acaiá IAC-474-19 & $9,73^{\mathrm{A}}$ & $8,10^{\mathrm{A}}$ & $6,80^{\mathrm{A}}$ & $8,21^{\mathrm{A}}$ & \\
\hline \multirow[t]{5}{*}{$\mathrm{N}$} & Icatu Amarelo IAC-3282 & $6,18^{\mathrm{A}}$ & $5,30^{\mathrm{A}}$ & $5,03^{\mathrm{A}}$ & $5,50^{\mathrm{A}}$ & 11,14 \\
\hline & Rubi MG-1192 & $6,43^{\mathrm{A}}$ & $4,00^{\mathrm{A}}$ & $4,23^{\mathrm{A}}$ & $4,88^{\mathrm{A}}$ & \\
\hline & Catuaí Vermelho IAC-99 & $4,80^{\mathrm{A}}$ & $4,45^{\mathrm{A}}$ & $3,73^{A}$ & $4,33^{\mathrm{A}}$ & \\
\hline & Média & $6,78^{\text {a }}$ & $5,46^{\mathrm{b}}$ & $4,94^{\mathrm{c}}$ & & \\
\hline & Acaiá IAC-474-19 & $13,13^{\mathrm{A}}$ & $9,60^{A}$ & $7,85^{\mathrm{A}}$ & $10,19^{\mathrm{A}}$ & \\
\hline \multirow[t]{5}{*}{$\mathrm{P}$} & Icatu Amarelo IAC-3282 & $5,95^{\mathrm{A}}$ & $5,18^{\mathrm{A}}$ & $6,00^{\mathrm{A}}$ & $5,71^{\mathrm{A}}$ & 25,42 \\
\hline & Rubi MG-1192 & $8,55^{\mathrm{A}}$ & $4,40^{\mathrm{A}}$ & $5,65^{\mathrm{A}}$ & $6,20^{\mathrm{A}}$ & \\
\hline & Catuaí Vermelho IAC-99 & $5,88^{\mathrm{A}}$ & $5,20^{\mathrm{A}}$ & $4,38^{\mathrm{A}}$ & $5,15^{\mathrm{A}}$ & \\
\hline & Média & $8,38^{a}$ & $6,09^{b}$ & $5,97^{c}$ & & \\
\hline & Acaiá IAC-474-19 & $7,03^{\mathrm{A}}$ & $7,08^{\mathrm{A}}$ & $5,28^{A}$ & $6,46^{\mathrm{A}}$ & \\
\hline \multirow[t]{5}{*}{$\mathrm{S}$} & Icatu Amarelo IAC-3282 & $4,25^{\mathrm{A}}$ & $4,55^{\mathrm{A}}$ & $4,03^{\mathrm{A}}$ & $4,28^{\mathrm{A}}$ & 16,50 \\
\hline & Rubi MG-1192 & $4,35^{\mathrm{A}}$ & $3,55^{\mathrm{A}}$ & $3,40^{\mathrm{A}}$ & $3,77^{\mathrm{A}}$ & \\
\hline & Catuaí Vermelho IAC-99 & $3,63^{A}$ & $3,80^{\mathrm{A}}$ & $3,25^{\mathrm{A}}$ & $3,56^{\mathrm{A}}$ & \\
\hline & Média & $4,81^{\mathrm{a}}$ & $4,74^{\mathrm{a}}$ & $3,99^{a}$ & & \\
\hline & Acaiá IAC-474-19 & $9,05^{\mathrm{A}}$ & $7,03^{\mathrm{A}}$ & $5,50^{\mathrm{A}}$ & $7,19^{\mathrm{A}}$ & \\
\hline \multirow[t]{5}{*}{ K } & Icatu Amarelo IAC-3282 & $5,70^{\mathrm{A}}$ & $4,53^{\mathrm{A}}$ & $4,20^{\mathrm{A}}$ & $4,81^{\mathrm{A}}$ & 18,88 \\
\hline & Rubi MG-1192 & $5,30^{\mathrm{A}}$ & $2,80^{\mathrm{A}}$ & $3,18^{\mathrm{A}}$ & $3,76^{\mathrm{A}}$ & \\
\hline & Catuaí Vermelho IAC-99 & $3,80^{\mathrm{A}}$ & $3,40^{\mathrm{A}}$ & $2,88^{\mathrm{A}}$ & $3,36^{\mathrm{A}}$ & \\
\hline & Média & $5,96^{\text {a }}$ & $4,44^{b}$ & $3,94^{b}$ & & \\
\hline & Acaiá IAC-474-19 & $15,15^{\mathrm{A}}$ & $13,40^{\mathrm{A}}$ & $14,08^{\mathrm{A}}$ & $14,21^{\mathrm{A}}$ & \\
\hline \multirow[t]{5}{*}{$\mathrm{Ca}$} & Icatu Amarelo IAC-3282 & $8,88^{\mathrm{A}}$ & $7,80^{\mathrm{A}}$ & $10,40^{\mathrm{A}}$ & $9,03^{\mathrm{A}}$ & 17,50 \\
\hline & Rubi MG-1192 & $7,60^{\mathrm{A}}$ & $7,28^{\mathrm{A}}$ & $7,15^{\mathrm{A}}$ & $7,34^{\mathrm{A}}$ & \\
\hline & Catuaí Vermelho IAC-99 & $6,68^{\mathrm{A}}$ & $6,50^{\mathrm{A}}$ & $5,50^{\mathrm{A}}$ & $6,23^{\mathrm{A}}$ & \\
\hline & Média & $9,58^{\mathrm{a}}$ & $8,74^{\mathrm{a}}$ & $9,28^{\mathrm{a}}$ & & \\
\hline & Acaiá IAC-474-19 & $9,38^{\mathrm{A}}$ & $8,08^{\mathrm{A}}$ & $6,90^{\mathrm{A}}$ & $8,12^{\mathrm{A}}$ & \\
\hline \multirow[t]{5}{*}{$\mathrm{Mg}$} & Icatu Amarelo IAC-3282 & $5,78^{\mathrm{A}}$ & $5,78^{\mathrm{A}}$ & $5,30^{\mathrm{A}}$ & $5,62^{\mathrm{A}}$ & 15,01 \\
\hline & Rubi MG-1192 & $5,70^{\mathrm{A}}$ & $4,05^{\mathrm{A}}$ & $4,60^{\mathrm{A}}$ & $4,78^{\mathrm{A}}$ & \\
\hline & Catuaí Vermelho IAC-99 & $4,68^{\mathrm{A}}$ & $4,18^{\mathrm{A}}$ & $4,40^{\mathrm{A}}$ & $4,42^{\mathrm{A}}$ & \\
\hline & Média & $6,38^{a}$ & $5,52^{b}$ & $5,30^{b}$ & & \\
\hline & Acaiá IAC-474-19 & $14,70^{\mathrm{A}}$ & $14,98^{\mathrm{A}}$ & $10,88^{\mathrm{A}}$ & $13,52^{\mathrm{A}}$ & \\
\hline \multirow[t]{5}{*}{$\mathrm{Cu}$} & Icatu Amarelo IAC-3282 & $8,33^{\mathrm{A}}$ & $10,05^{\mathrm{A}}$ & $8,53^{\mathrm{A}}$ & $8,97^{\mathrm{A}}$ & 16,49 \\
\hline & Rubi MG-1192 & $9,75^{\mathrm{A}}$ & $7,95^{\mathrm{A}}$ & $9,18^{\mathrm{A}}$ & $8,96^{\mathrm{A}}$ & \\
\hline & Catuaí Vermelho IAC-99 & $8,03^{A}$ & $8,75^{\mathrm{A}}$ & $6,88^{\mathrm{A}}$ & $7,88^{\mathrm{A}}$ & \\
\hline & Média & $10,20^{\mathrm{a}}$ & $10,43^{\mathrm{a}}$ & $8,86^{\mathrm{a}}$ & & \\
\hline & Acaiá IAC-474-19 & $7,23^{\mathrm{A}}$ & $7,35^{\mathrm{A}}$ & $7,18^{\mathrm{A}}$ & $7,25^{\mathrm{A}}$ & \\
\hline \multirow[t]{5}{*}{ Zn } & Icatu Amarelo IAC-3282 & $5,25^{\mathrm{A}}$ & $5,08^{A}$ & $5,45^{\mathrm{A}}$ & $5,26^{A}$ & 18,48 \\
\hline & Rubi MG-1192 & $5,43^{\mathrm{A}}$ & $4,70^{\mathrm{A}}$ & $5,83^{\mathrm{A}}$ & $5,32^{\mathrm{A}}$ & \\
\hline & Catuaí Vermelho IAC-99 & $4,08^{\mathrm{A}}$ & $5,90^{\mathrm{A}}$ & $4,98^{\mathrm{A}}$ & $4,98^{\mathrm{A}}$ & \\
\hline & Média & $5,49^{\mathrm{a}}$ & $5,76^{a}$ & $5,86^{\mathrm{a}}$ & & \\
\hline & Acaiá IAC-474-19 & $3,15^{\mathrm{A}}$ & $3,58^{\mathrm{A}}$ & $2,85^{\mathrm{A}}$ & $3,19^{\mathrm{A}}$ & \\
\hline \multirow[t]{4}{*}{ B } & Icatu Amarelo IAC-3282 & $3,00^{\mathrm{A}}$ & $1,80^{\mathrm{A}}$ & $2,08^{\mathrm{A}}$ & $2,29^{\mathrm{A}}$ & 57,23 \\
\hline & Rubi MG-1192 & $2,18^{\mathrm{A}}$ & $1,45^{\mathrm{A}}$ & $0,85^{\mathrm{A}}$ & $1,49^{\mathrm{A}}$ & \\
\hline & Catuaí Vermelho IAC-99 & $2,33^{\mathrm{A}}$ & $1,25^{\mathrm{A}}$ & $0,70^{\mathrm{A}}$ & $1,43^{\mathrm{A}}$ & \\
\hline & Média & $2,66^{\mathrm{a}}$ & $2,02^{\mathrm{a}}$ & $1,62^{\mathrm{a}}$ & & \\
\hline
\end{tabular}

Médias seguidas pela mesma letra maiúscula na coluna e minúscula na linha não diferem estatisticamente entre si a $10 \%$, pelo teste de Tukey.

${ }^{2 /} \mathrm{ANC}=\frac{\text { Conteúdo de nutriente no caule }}{\text { Conteúdo de nutriente na parte aérea }} \times 100$, em \% para macro e micronutrientes. 
Diferenças entre cultivares na alocação relativa de nutrientes em ramos só se verificaram no nível baixo de fertilização para P e Ca e no nível normal para N (Tabela 5). O cultivar Rubi MG 1192 alocou mais P nos ramos e foi intermediário na alocação de Ca nesse órgão, tendo o inverso ocorrido para o cultivar Catuaí Vermelho IAC 99, o qual alocou mais Ca e teve alocação intermediária de P nos ramos. Os cultivares Acaiá IAC 47419 e Icatu Amarelo IAC 3282 alocaram menos P nos ramos. No nível normal, o cultivar Catuaí Vermelho IAC-99 alocou maior quantidade de $\mathrm{N}$ nos ramos, enquanto a menor alocação desse nutriente nos ramos ocorreu para os cultivares Acaiá IAC-474-19 e Rubi MG-1192.

Tabela 5 - Alocação relativa de nutrientes em $\operatorname{ramos}^{3}$ de quatro cultivares de café arábica, submetidos a três níveis de adubação

\begin{tabular}{|c|c|c|c|c|c|c|}
\hline Nutriente & Cultivar & $\frac{\text { Nível de Adubação }}{\text { Baixo }}$ & $\begin{array}{c}\text { Média } \\
\text { Normal }\end{array}$ & $\begin{array}{c}\text { CV (\%) } \\
\text { Alto }\end{array}$ & Média & CV (\%) \\
\hline & Acaiá IAC-474-19 & $13,50^{\mathrm{A}}$ & $14,50^{\text {в }}$ & $14,00^{\mathrm{A}}$ & $14,00^{\mathrm{A}}$ & \\
\hline \multirow[t]{5}{*}{$\mathrm{N}$} & Icatu Amarelo IAC-3282 & $15,00^{\mathrm{A}}$ & 15,25 Ав & $13,75^{\mathrm{A}}$ & $14,67^{\mathrm{A}}$ & 15,50 \\
\hline & Rubi MG-1192 & $15,75^{\mathrm{A}}$ & $14,00^{\text {в }}$ & $13,75^{\mathrm{A}}$ & $14,50^{\mathrm{A}}$ & \\
\hline & Catuaí Vermelho IAC-99 & $16,00^{\mathrm{A}}$ & $18,50^{\mathrm{A}}$ & $14,75^{\mathrm{A}}$ & $16,42^{\mathrm{A}}$ & \\
\hline & Média & $15,06^{\mathrm{a}}$ & $15,56^{\mathrm{a}}$ & $14,06^{\mathrm{a}}$ & & \\
\hline & Acaiá IAC-474-19 & $13,75^{\text {в }}$ & $15,75^{\mathrm{A}}$ & $14,00^{\mathrm{A}}$ & $14,50^{\mathrm{A}}$ & \\
\hline \multirow[t]{5}{*}{$\mathrm{P}$} & Icatu Amarelo IAC-3282 & $13,00^{\text {в }}$ & $13,00^{\mathrm{A}}$ & $12,50^{\mathrm{A}}$ & $12,83^{\mathrm{A}}$ & 24,26 \\
\hline & Rubi MG-1192 & $20,25^{\mathrm{A}}$ & $13,75^{\mathrm{A}}$ & $11,75^{\mathrm{A}}$ & $15,25^{\mathrm{A}}$ & \\
\hline & Catuaí Vermelho IAC-99 & 15,25 АВ & $16,25^{\mathrm{A}}$ & $10,50^{\mathrm{A}}$ & $14,00^{\mathrm{A}}$ & \\
\hline & Média & $15,56^{\mathrm{a}}$ & $14,69^{\text {a }}$ & $12,19^{\mathrm{a}}$ & & \\
\hline & Acaiá IAC-474-19 & $16,50^{\mathrm{A}}$ & $19,75^{\mathrm{A}}$ & $18,50^{\mathrm{A}}$ & $18,25^{\mathrm{A}}$ & \\
\hline \multirow[t]{5}{*}{ S } & Icatu Amarelo IAC-3282 & $18,25^{\mathrm{A}}$ & $19,75^{\mathrm{A}}$ & $18,00^{\mathrm{A}}$ & $18,67^{\mathrm{A}}$ & 12,80 \\
\hline & Rubi MG-1192 & $18,25^{\mathrm{A}}$ & $17,50^{\mathrm{A}}$ & $16,00^{\mathrm{A}}$ & $17,25^{\mathrm{A}}$ & \\
\hline & Catuaí Vermelho IAC-99 & $18,25^{\mathrm{A}}$ & $21,50^{\mathrm{A}}$ & $16,50^{\mathrm{A}}$ & $18,75^{\mathrm{A}}$ & \\
\hline & Média & $17,81^{\text {a }}$ & $19,63^{\text {a }}$ & $17,25^{\mathrm{a}}$ & & \\
\hline & Acaiá IAC-474-19 & $15,00^{\mathrm{A}}$ & $17,50^{\mathrm{A}}$ & $15,50^{\mathrm{A}}$ & $16,00^{\mathrm{A}}$ & \\
\hline \multirow[t]{5}{*}{ K } & Icatu Amarelo IAC-3282 & $17,50^{\mathrm{A}}$ & $18,00^{\mathrm{A}}$ & $14,50^{\mathrm{A}}$ & $16,67^{\mathrm{A}}$ & 18,70 \\
\hline & Rubi MG-1192 & $17,25^{\mathrm{A}}$ & $14,00^{\mathrm{A}}$ & $11,75^{\mathrm{A}}$ & $14,33^{\mathrm{A}}$ & \\
\hline & Catuaí Vermelho IAC-99 & $17,00^{\mathrm{A}}$ & $19,00^{\mathrm{A}}$ & $13,50^{\mathrm{A}}$ & $16,50^{\mathrm{A}}$ & \\
\hline & Média & $16,69^{\text {a }}$ & $17,13^{\text {a }}$ & $13,81^{b}$ & & \\
\hline & Acaiá IAC-474-19 & $22,50^{\text {в }}$ & $23,50^{\mathrm{A}}$ & $23,00^{\mathrm{A}}$ & $23,00^{\mathrm{A}}$ & \\
\hline \multirow[t]{5}{*}{$\mathrm{Ca}$} & Icatu Amarelo IAC-3282 & 25,75 Ав & $23,75^{, A}$ & $20,50^{\mathrm{A}}$ & $23,33^{A}$ & 13,83 \\
\hline & Rubi MG-1192 & 24,75 АВ & $22,25^{\mathrm{A}}$ & $22,00^{\mathrm{A}}$ & $23,00^{\mathrm{A}}$ & \\
\hline & Catuaí Vermelho IAC-99 & $29,00^{\mathrm{A}}$ & $26,25^{\mathrm{A}}$ & $24,50^{\mathrm{A}}$ & $26,58^{A}$ & \\
\hline & Média & $25,50^{\text {a }}$ & $23,94^{\mathrm{a}}$ & $22,50^{\mathrm{a}}$ & & \\
\hline & Acaiá IAC-474-19 & $14,50^{\mathrm{A}}$ & $16,25^{\mathrm{A}}$ & $17,50^{\mathrm{A}}$ & $16,08^{\mathrm{A}}$ & \\
\hline \multirow[t]{5}{*}{$\mathrm{Mg}$} & Icatu Amarelo IAC-3282 & $16,00^{\mathrm{A}}$ & $16,25^{\mathrm{A}}$ & $17,00^{\mathrm{A}}$ & $16,42^{\mathrm{A}}$ & 15,07 \\
\hline & Rubi MG-1192 & $16,75^{\mathrm{A}}$ & $16,00^{\mathrm{A}}$ & $19,25^{\mathrm{A}}$ & $17,33^{\mathrm{A}}$ & \\
\hline & Catuaí Vermelho IAC-99 & $17,25^{\mathrm{A}}$ & $19,00^{\mathrm{A}}$ & $19,25^{\mathrm{A}}$ & $18,50^{\mathrm{A}}$ & \\
\hline & Média & $16,13^{\text {a }}$ & $16,88^{\text {a }}$ & $18,25^{\mathrm{a}}$ & & \\
\hline & Acaiá IAC-474-19 & $27,25^{\mathrm{A}}$ & $37,75^{\mathrm{A}}$ & $37,50^{\mathrm{A}}$ & $34,17^{\mathrm{A}}$ & \\
\hline \multirow[t]{5}{*}{$\mathrm{Cu}$} & Icatu Amarelo IAC-3282 & $25,00^{\mathrm{A}}$ & $30,50^{\mathrm{A}}$ & $33,25^{\mathrm{A}}$ & $29,58^{A}$ & 17,57 \\
\hline & Rubi MG-1192 & $31,00^{\mathrm{A}}$ & $34,00^{\mathrm{A}}$ & $34,25^{\mathrm{A}}$ & $33,08^{A}$ & \\
\hline & Catuaí Vermelho IAC-99 & $32,50^{\mathrm{A}}$ & $36,75^{\mathrm{A}}$ & $34,50^{\mathrm{A}}$ & $34,58^{A}$ & \\
\hline & Média & $28,94^{b}$ & $34,75^{\text {a }}$ & $34,88^{a}$ & & \\
\hline & Acaiá IAC-474-19 & $43,50^{\mathrm{A}}$ & $53,00^{\mathrm{A}}$ & $58,75^{\mathrm{A}}$ & $51,75^{\mathrm{A}}$ & \\
\hline \multirow[t]{5}{*}{$\mathrm{Zn}$} & Icatu Amarelo IAC-3282 & $42,75^{\mathrm{A}}$ & $48,75^{\mathrm{A}}$ & $57,25^{\mathrm{A}}$ & $49,58^{A}$ & 12,57 \\
\hline & Rubi MG-1192 & $49,00^{\mathrm{A}}$ & $53,25^{\mathrm{A}}$ & $57,75^{\mathrm{A}}$ & $53,33^{\mathrm{A}}$ & \\
\hline & Catuaí Vermelho IAC-99 & $45,00^{\mathrm{A}}$ & $52,25^{\mathrm{A}}$ & $56,00^{\mathrm{A}}$ & $51,08^{A}$ & \\
\hline & Média & $45,06^{c}$ & $51,81^{\text {b }}$ & $57,44^{\text {a }}$ & & \\
\hline & Acaiá IAC-474-19 & $9,25^{\mathrm{A}}$ & $17,75^{\mathrm{A}}$ & $17,25^{\mathrm{A}}$ & $14,75^{\mathrm{A}}$ & \\
\hline \multirow[t]{4}{*}{ B } & Icatu Amarelo IAC-3282 & $11,50^{\mathrm{A}}$ & $16,75^{\mathrm{A}}$ & $15,50^{\mathrm{A}}$ & $14,58^{\mathrm{A}}$ & 35,92 \\
\hline & Rubi MG-1192 & $9,75^{\mathrm{A}}$ & $12,75^{\mathrm{A}}$ & $13,75^{\mathrm{A}}$ & $12,08^{\mathrm{A}}$ & \\
\hline & Catuaí Vermelho IAC-99 & $10,50^{\mathrm{A}}$ & $14,00^{\mathrm{A}}$ & $12,75^{\mathrm{A}}$ & $12,42^{\mathrm{A}}$ & \\
\hline & Média & $10,25^{b}$ & $15,31^{\text {a }}$ & $14,81^{\text {a }}$ & & \\
\hline
\end{tabular}

Médias seguidas pela mesma letra maiúscula na coluna e minúscula na linha não diferem estatisticamente entre si a $10 \%$, pelo teste de Tukey.

3/ $\mathrm{ANR}=\frac{\text { Conteúdo de nutriente nos ramos }}{\text { Conteúdo de nutriente na parte aérea }} \times 100$, em \% para macro e micronutrientes. 
A alocação relativa de nutrientes em folhas (Tabela 6) expressa a razão entre o conteúdo de nutrientes nas folhas e na parte aérea. No menor nível de fertilização o Rubi MG 1192 apresentou maior alocação de nutrientes em folhas, enquanto o Acaiá IAC 47419 manteve-se numa posição intermediária. Icatu Amarelo IAC 3282 e Catuaí Ver- melho IAC 99 exibiram menor alocação de nutrientes nesse órgão. No nível normal de adubação, os quatro cultivares exibiram o mesmo comportamento, não havendo diferenças entre eles quanto à alocação de nutrientes nas folhas. No nível mais alto de adubação, verificou-se diferença entre os cultivares na alocação de K e Mg nas

Tabela 6 - Alocação relativa de nutrientes em folhas ${ }^{4}$ de quatro cultivares de café arábica, submetidos a três níveis de adubação

\begin{tabular}{|c|c|c|c|c|c|c|}
\hline \multirow{2}{*}{ Nutriente } & \multirow{2}{*}{ Cultivar } & \multicolumn{3}{|c|}{ Nível de Adubação } & \multirow{2}{*}{ Média } & \multirow{2}{*}{ CV (\%) } \\
\hline & & Baixo & Normal & Alto & & \\
\hline & Acaiá IAC-474-19 & $40,25 \mathrm{AB}$ & $49,25^{\mathrm{A}}$ & $43,75^{\mathrm{A}}$ & $44,42^{\mathrm{A}}$ & \\
\hline \multirow[t]{5}{*}{$\mathrm{N}$} & Icatu Amarelo IAC-3282 & $33,00^{\text {в }}$ & $38,25^{\mathrm{A}}$ & $41,00^{\mathrm{A}}$ & $37,42^{\mathrm{A}}$ & 21,21 \\
\hline & Rubi MG-1192 & $45,00^{\mathrm{A}}$ & $38,50^{\mathrm{A}}$ & $32,25^{\mathrm{A}}$ & $38,58^{A}$ & \\
\hline & Catuaí Vermelho IAC-99 & $31,75^{\text {в }}$ & $37,50^{\mathrm{A}}$ & $29,25^{\mathrm{A}}$ & $32,83^{\mathrm{A}}$ & \\
\hline & Média & $37,50^{\mathrm{a}}$ & $40,88^{a}$ & $36,56^{\mathrm{a}}$ & & \\
\hline & Acaiá IAC-474-19 & $27,50^{\mathrm{A}}$ & $38,25^{\mathrm{A}}$ & $33,50^{\mathrm{A}}$ & $33,08^{A}$ & \\
\hline \multirow[t]{5}{*}{$\mathrm{P}$} & Icatu Amarelo IAC-3282 & $24,50^{\mathrm{A}}$ & $31,00^{\mathrm{A}}$ & $33,75^{\mathrm{A}}$ & $29,83^{\mathrm{A}}$ & 23,13 \\
\hline & Rubi MG-1192 & $33,25^{\mathrm{A}}$ & $30,75^{\mathrm{A}}$ & $26,75^{\mathrm{A}}$ & $30,25^{\mathrm{A}}$ & \\
\hline & Catuaí Vermelho IAC-99 & $23,50^{\mathrm{A}}$ & $31,50^{\mathrm{A}}$ & $22,50^{\mathrm{A}}$ & $25,83^{A}$ & \\
\hline & Média & $27,25^{a}$ & $32,88^{a}$ & $29,13^{\text {a }}$ & & \\
\hline & Acaiá IAC-474-19 & 36,00 АВ & $42,25^{\mathrm{A}}$ & $39,75^{\mathrm{A}}$ & $39,33^{\mathrm{A}}$ & \\
\hline \multirow[t]{5}{*}{ S } & Icatu Amarelo IAC-3282 & $27,50^{\text {в }}$ & $31,75^{\mathrm{A}}$ & $36,75^{\mathrm{A}}$ & $32,00^{\mathrm{A}}$ & 24,68 \\
\hline & Rubi MG-1192 & $43,75^{\mathrm{A}}$ & $34,50^{\mathrm{A}}$ & $34,50^{\mathrm{A}}$ & $37,58^{\mathrm{A}}$ & \\
\hline & Catuaí Vermelho IAC-99 & $28,25^{\text {в }}$ & $33,25^{\mathrm{A}}$ & $26,50^{\mathrm{A}}$ & $29,33^{\mathrm{A}}$ & \\
\hline & Média & $33,88^{\mathrm{a}}$ & $35,44^{\mathrm{a}}$ & $34,38^{\mathrm{a}}$ & & \\
\hline & Acaiá IAC-474-19 & 31,75 Ав & $43,50^{\mathrm{A}}$ & $39,50^{\mathrm{A}}$ & $38,25^{\mathrm{A}}$ & \\
\hline \multirow[t]{5}{*}{ K } & Icatu Amarelo IAC-3282 & 28,00 АВ & $33,25^{\mathrm{A}}$ & 37,75 АВ & $33,00^{\mathrm{A}}$ & 24,38 \\
\hline & Rubi MG-1192 & $40,75^{\mathrm{A}}$ & $32,00^{\mathrm{A}}$ & 26,75 АВ & $33,17^{\mathrm{A}}$ & \\
\hline & Catuaí Vermelho IAC-99 & $26,00^{\text {в }}$ & $34,25^{\mathrm{A}}$ & $25,00^{\text {в }}$ & $28,42^{\mathrm{A}}$ & \\
\hline & Média & $31,63^{\mathrm{a}}$ & $35,75^{a}$ & $32,25^{\mathrm{a}}$ & & \\
\hline & Acaiá IAC-474-19 & 50,50 Ав & $54,50^{\mathrm{A}}$ & $53,25^{\mathrm{A}}$ & $52,75^{\mathrm{A}}$ & \\
\hline \multirow[t]{5}{*}{$\mathrm{Ca}$} & Icatu Amarelo IAC-3282 & $46,25^{\text {в }}$ & $52,50^{\mathrm{A}}$ & $53,75^{\mathrm{A}}$ & $50,83^{\mathrm{A}}$ & 9,91 \\
\hline & Rubi MG-1192 & $57,00^{\mathrm{A}}$ & $55,75^{\mathrm{A}}$ & $52,00^{\mathrm{A}}$ & $54,92^{\mathrm{A}}$ & \\
\hline & Catuaí Vermelho IAC-99 & 48,25 АВ & $53,75^{\mathrm{A}}$ & $51,50^{\mathrm{A}}$ & $51,17^{\mathrm{A}}$ & \\
\hline & Média & $50,50^{\mathrm{a}}$ & $54,13^{a}$ & $52,63^{\text {a }}$ & & \\
\hline & Acaiá IAC-474-19 & 48,00 АВ & $57,00^{\mathrm{A}}$ & $52,75^{\mathrm{A}}$ & $52,58^{A}$ & \\
\hline \multirow[t]{5}{*}{$\mathrm{Mg}$} & Icatu Amarelo IAC-3282 & $41,00^{\text {в }}$ & $49,75^{\mathrm{A}}$ & 51,75 Ав & $47,50^{\mathrm{A}}$ & 14,69 \\
\hline & Rubi MG-1192 & $53,75^{\mathrm{A}}$ & $52,00^{\mathrm{A}}$ & 48,75 АВ & $51,50^{\mathrm{A}}$ & \\
\hline & Catuaí Vermelho IAC-99 & $42,50 \mathrm{AB}$ & $51,50^{\mathrm{A}}$ & $39,50^{\text {в }}$ & $44,50^{\mathrm{A}}$ & \\
\hline & Média & $46,31^{\mathrm{a}}$ & $52,56^{\mathrm{a}}$ & $48,19^{a}$ & & \\
\hline & Acaiá IAC-474-19 & 22,50 Ав & $16,25^{\mathrm{A}}$ & $16,25^{\mathrm{A}}$ & $18,33^{\mathrm{A}}$ & \\
\hline \multirow{5}{*}{$\mathrm{Cu}$} & Icatu Amarelo IAC-3282 & $15,75^{\text {в }}$ & $14,25^{\mathrm{A}}$ & $18,00^{\mathrm{A}}$ & $16,00^{\mathrm{A}}$ & 27,75 \\
\hline & Rubi MG-1192 & $26,00^{\mathrm{A}}$ & $13,25^{\mathrm{A}}$ & $12,50^{\mathrm{A}}$ & $17,25^{\mathrm{A}}$ & \\
\hline & Catuaí Vermelho IAC-99 & $15,75^{\text {в }}$ & $13,00^{\mathrm{A}}$ & $12,00^{\mathrm{A}}$ & $13,58^{\mathrm{A}}$ & \\
\hline & Média & $20,00^{a}$ & $14,19^{b}$ & $14,69^{\mathrm{b}}$ & & \\
\hline & Acaiá IAC-474-19 & $24,25^{\mathrm{A}}$ & $26,00^{\mathrm{A}}$ & $17,50^{\mathrm{A}}$ & $22,58^{A}$ & \\
\hline \multirow[t]{5}{*}{ Zn } & Icatu Amarelo IAC-3282 & $17,50^{\mathrm{A}}$ & $22,25^{\mathrm{A}}$ & $16,25^{\mathrm{A}}$ & $18,67^{\mathrm{A}}$ & 22,95 \\
\hline & Rubi MG-1192 & $22,75^{\mathrm{A}}$ & $22,00^{\mathrm{A}}$ & $14,00^{\mathrm{A}}$ & $19,58^{\mathrm{A}}$ & \\
\hline & Catuaí Vermelho IAC-99 & $17,00^{\mathrm{A}}$ & $21,50^{\mathrm{A}}$ & $16,00^{\mathrm{A}}$ & $18,17^{\mathrm{A}}$ & \\
\hline & Média & $20,38^{a}$ & $22,94^{\text {a }}$ & $15,94^{b}$ & & \\
\hline & Acaiá IAC-474-19 & $62,00^{\mathrm{A}}$ & $68,00^{\mathrm{A}}$ & $64,00^{\mathrm{A}}$ & $64,67^{\mathrm{A}}$ & \\
\hline \multirow[t]{4}{*}{ B } & Icatu Amarelo IAC-3282 & $55,25^{A}$ & $62,25^{\mathrm{A}}$ & $68,75^{\mathrm{A}}$ & $62,08^{A}$ & 15,59 \\
\hline & Rubi MG-1192 & $64,25^{\mathrm{A}}$ & $65,00^{\mathrm{A}}$ & $67,, 50^{\mathrm{A}}$ & $65,58^{\mathrm{A}}$ & \\
\hline & Catuaí Vermelho IAC-99 & $52,50^{\mathrm{A}}$ & $64,50^{\mathrm{A}}$ & $57,26^{\mathrm{A}}$ & $58,08^{\mathrm{A}}$ & \\
\hline & Média & $58,50^{\text {a }}$ & $64,94^{\mathrm{a}}$ & $64,38^{\text {a }}$ & & \\
\hline
\end{tabular}

Médias seguidas pela mesma letra maiúscula na coluna e minúscula na linha não diferem estatisticamente entre si a $10 \%$, pelo teste de Tukey.

4/ $\mathrm{ANF}=\frac{\text { Conteúdo de nutriente nas folhas }}{\text { Conteúdo de nutriente na parte aérea }} \times 100$, em \% para macro e micronutrientes. 
folhas, mantendo-se o mesmo padrão de comportamento verificado no menor nível.

Os cultivares não exibiram diferenças quanto à alocação relativa de nutrientes nos frutos (Tabela 7).

O caule apresentou menor conteúdo de todos os nutrientes em relação aos demais órgãos da parte aérea. Os frutos acumularam maior quantidade de $\mathrm{P}, \mathrm{S}$, $\mathrm{K}$ e $\mathrm{Cu}$, enquanto as folhas detiveram maior conteúdo de $\mathrm{N}$, Ca, Mg e B. Por sua vez, o maior conteúdo de $Z n$ verificou-se nos ramos (Tabelas 4 a 7 ).

O cultivar Rubi MG-1192 alocou mais N, S, K, Ca, Mg e $\mathrm{Cu}$ nas folhas e apresenta tendência em alocar menor

Tabela 7 - Alocação relativa de nutrientes em frutos ${ }^{5}$ de quatro cultivares de café arábica, submetidos a três níveis de adubação

\begin{tabular}{|c|c|c|c|c|c|c|}
\hline \multirow{2}{*}{ Nutriente } & \multirow{2}{*}{ Cultivar } & \multicolumn{3}{|c|}{ Nível de Adubação } & \multirow{2}{*}{ Média } & \multirow{2}{*}{ CV (\%) } \\
\hline & & Baixo & Normal & Alto & & \\
\hline & Acaiá IAC-474-19 & $36,52^{\mathrm{A}}$ & $28,15^{\mathrm{A}}$ & $35,45^{\mathrm{A}}$ & $33,37^{\mathrm{A}}$ & \\
\hline \multirow[t]{5}{*}{$\mathrm{N}$} & Icatu Amarelo IAC-3282 & $45,82^{\mathrm{A}}$ & $41,20^{\mathrm{A}}$ & $40,22^{\mathrm{A}}$ & $42,41^{\mathrm{A}}$ & 44,68 \\
\hline & Rubi MG-1192 & $32,82^{\mathrm{A}}$ & $43,50^{\mathrm{A}}$ & $49,77^{\mathrm{A}}$ & $42,04^{\mathrm{A}}$ & \\
\hline & Catuaí Vermelho IAC-99 & $47,45^{\mathrm{A}}$ & $39,55^{\mathrm{A}}$ & $52,27^{\mathrm{A}}$ & $46,42^{\mathrm{A}}$ & \\
\hline & Média & $40,66^{\mathrm{a}}$ & $38,10^{\mathrm{a}}$ & $44,44^{\mathrm{a}}$ & & \\
\hline & Acaiá IAC-474-19 & $45,62^{\mathrm{A}}$ & $36,40^{\mathrm{A}}$ & $44,65^{\mathrm{A}}$ & $42,23^{\mathrm{A}}$ & \\
\hline \multirow[t]{5}{*}{$\mathrm{P}$} & Icatu Amarelo IAC-3282 & $56,30^{\mathrm{A}}$ & $50,82^{\mathrm{A}}$ & $47,75^{\mathrm{A}}$ & $51,63^{\mathrm{A}}$ & 46,29 \\
\hline & Rubi MG-1192 & $37,95^{\mathrm{A}}$ & $51,10^{\mathrm{A}}$ & $55,85^{\mathrm{A}}$ & $48,30^{\mathrm{A}}$ & \\
\hline & Catuaí Vermelho IAC-99 & $55,37^{\mathrm{A}}$ & $47,05^{\mathrm{A}}$ & $62,62^{\mathrm{A}}$ & $55,02^{\mathrm{A}}$ & \\
\hline & Média & $48,81^{\mathrm{a}}$ & $46,34^{\mathrm{a}}$ & $52,71^{\mathrm{a}}$ & & \\
\hline & Acaiá IAC-474-19 & $40,47^{\mathrm{A}}$ & $30,92^{\mathrm{A}}$ & $36,47^{\mathrm{A}}$ & $35,96^{\mathrm{A}}$ & \\
\hline \multirow[t]{5}{*}{ S } & Icatu Amarelo IAC-3282 & $50,00^{\mathrm{A}}$ & $43,95^{\mathrm{A}}$ & $41,2^{\mathrm{A}}$ & $45,05^{\mathrm{A}}$ & 42,58 \\
\hline & Rubi MG-1192 & $33,65^{\mathrm{A}}$ & $44,45^{\mathrm{A}}$ & $46,10^{\mathrm{A}}$ & $41,40^{\mathrm{A}}$ & \\
\hline & Catuaí Vermelho IAC-99 & $49,87^{\mathrm{A}}$ & $41,45^{\mathrm{A}}$ & $53,75^{\mathrm{A}}$ & $48,36^{\mathrm{A}}$ & \\
\hline & Média & $43,50^{\mathrm{a}}$ & $40,19^{\mathrm{a}}$ & $44,38^{\mathrm{a}}$ & & \\
\hline & Acaiá IAC-474-19 & $44,20^{\mathrm{A}}$ & $31,97^{\mathrm{A}}$ & $39,50^{\mathrm{A}}$ & $38,56^{\mathrm{A}}$ & \\
\hline \multirow[t]{5}{*}{ K } & Icatu Amarelo IAC-3282 & $48,80^{\mathrm{A}}$ & $44,22^{\mathrm{A}}$ & $43,55^{\mathrm{A}}$ & $45,52^{\mathrm{A}}$ & 44,77 \\
\hline & Rubi MG-1192 & $36,70^{\mathrm{A}}$ & $51,20^{\mathrm{A}}$ & $58,32^{\mathrm{A}}$ & $48,74^{\mathrm{A}}$ & \\
\hline & Catuaí Vermelho IAC-99 & $53,20^{\mathrm{A}}$ & $43,35^{\mathrm{A}}$ & $58,62^{\mathrm{A}}$ & $51,72^{\mathrm{A}}$ & \\
\hline & Média & $45,72^{\mathrm{a}}$ & $42,68^{\mathrm{a}}$ & $50,00^{\mathrm{a}}$ & & \\
\hline & Acaiá IAC-474-19 & $11,85^{\mathrm{A}}$ & $8,60^{\mathrm{A}}$ & $9,67^{\mathrm{A}}$ & $10,04^{\mathrm{A}}$ & \\
\hline \multirow[t]{5}{*}{ Ca } & Icatu Amarelo IAC-3282 & $19,12^{\mathrm{A}}$ & $15,95^{\mathrm{A}}$ & $15,35^{\mathrm{A}}$ & $16,81^{\mathrm{A}}$ & 55,96 \\
\hline & Rubi MG-1192 & $10,65^{\mathrm{A}}$ & $14,72^{\mathrm{A}}$ & $18,85^{\mathrm{A}}$ & $14,74^{\mathrm{A}}$ & \\
\hline & Catuaí Vermelho IAC-99 & $16,07^{\mathrm{A}}$ & $13,50^{\mathrm{A}}$ & $18,50^{\mathrm{A}}$ & $16,02^{\mathrm{A}}$ & \\
\hline & Média & $14,42^{\mathrm{a}}$ & $13,19^{\mathrm{a}}$ & $15,59^{a}$ & & \\
\hline & Acaiá IAC-474-19 & $28,12^{\mathrm{A}}$ & $18,67^{\mathrm{A}}$ & $22,85^{\mathrm{A}}$ & $23,22^{\mathrm{A}}$ & \\
\hline \multirow[t]{5}{*}{$\mathrm{Mg}$} & Icatu Amarelo IAC-3282 & $37,22^{\mathrm{A}}$ & $28,22^{\mathrm{A}}$ & $25,95^{\mathrm{A}}$ & $30,46^{\mathrm{A}}$ & 44,38 \\
\hline & Rubi MG-1192 & $23,80^{\mathrm{A}}$ & $27,95^{\mathrm{A}}$ & $27,40^{\mathrm{A}}$ & $26,39^{\mathrm{A}}$ & \\
\hline & Catuaí Vermelho IAC-99 & $35,57^{\mathrm{A}}$ & $25,32^{\mathrm{A}}$ & $36,85^{\mathrm{A}}$ & $32,58^{\mathrm{A}}$ & \\
\hline & Média & $31,18^{\mathrm{a}}$ & $25,04^{\mathrm{a}}$ & $28,26^{\mathrm{a}}$ & & \\
\hline & Acaiá IAC-474-19 & $35,55^{\mathrm{A}}$ & $31,02^{\mathrm{A}}$ & $35,37^{\mathrm{A}}$ & $33,98^{\mathrm{A}}$ & \\
\hline \multirow[t]{5}{*}{$\mathrm{Cu}$} & Icatu Amarelo IAC-3282 & $50,92^{\mathrm{A}}$ & $45,20^{\mathrm{A}}$ & $40,22^{\mathrm{A}}$ & $45,45^{\mathrm{A}}$ & 47,23 \\
\hline & Rubi MG-1192 & $33,25^{\mathrm{A}}$ & $44,80^{\mathrm{A}}$ & $44,07^{\mathrm{A}}$ & $40,71^{\mathrm{A}}$ & \\
\hline & Catuaí Vermelho IAC-99 & $43,72^{\mathrm{A}}$ & $41,50^{\mathrm{A}}$ & $46,62^{\mathrm{A}}$ & $43,96^{\mathrm{A}}$ & \\
\hline & Média & $40,86^{\mathrm{a}}$ & $40,63^{\mathrm{a}}$ & $41,57^{\mathrm{a}}$ & & \\
\hline & Acaiá IAC-474-19 & $25,02^{\mathrm{A}}$ & $13,65^{\mathrm{A}}$ & $16,57^{\mathrm{A}}$ & $18,42^{\mathrm{A}}$ & \\
\hline \multirow[t]{5}{*}{$\mathrm{Zn}$} & Icatu Amarelo IAC-3282 & $34,50^{\mathrm{A}}$ & $23,92^{\mathrm{A}}$ & $21,05^{\mathrm{A}}$ & $26,49^{\mathrm{A}}$ & 52,08 \\
\hline & Rubi MG-1192 & $22,82^{\mathrm{A}}$ & $20,05^{\mathrm{A}}$ & $22,42^{\mathrm{A}}$ & $21,77^{\mathrm{A}}$ & \\
\hline & Catuaí Vermelho IAC-99 & $33,92^{\mathrm{A}}$ & $20,35^{\mathrm{A}}$ & $23,02^{\mathrm{A}}$ & $25,77^{\mathrm{A}}$ & \\
\hline & Média & $29,07^{\mathrm{a}}$ & $19,49^{\mathrm{a}}$ & $20,76^{a}$ & & \\
\hline & Acaiá IAC-474-19 & $25,60^{\mathrm{A}}$ & $10,67^{\mathrm{A}}$ & $15,90^{\mathrm{A}}$ & $17,39^{\mathrm{A}}$ & \\
\hline \multirow[t]{4}{*}{ B } & Icatu Amarelo IAC-3282 & $30,25^{\mathrm{A}}$ & $19,20^{\mathrm{A}}$ & $13,67^{\mathrm{A}}$ & $21,05^{\mathrm{A}}$ & 79,85 \\
\hline & Rubi MG-1192 & $23,82^{\mathrm{A}}$ & $20,80^{\mathrm{A}}$ & $17,90^{\mathrm{A}}$ & $20,85^{\mathrm{A}}$ & \\
\hline & Catuaí Vermelho IAC-99 & $34,67^{\mathrm{A}}$ & $20,25^{\mathrm{A}}$ & $80,83^{\mathrm{A}}$ & $28,07^{\mathrm{A}}$ & \\
\hline & Média & $28,59^{a}$ & $17,73^{\mathrm{a}}$ & $19,19^{\mathrm{a}}$ & & \\
\hline
\end{tabular}


quantidade de nutrientes em frutos quando cultivado com baixo nível de adubação (Tabelas 6 e 7, respectivamente), o que resultaria em alta eficiência de produção de frutos para esse cultivar em tais condições. Nesse caso, a eficiência de produção de raízes parece ser o fator limitante da eficiência de produção de frutos.

Considerando a média de alocação relativa de nutrientes nos frutos para os quatro cultivares, no nível normal de adubação (Tabela 7), verificou-se que eles possuem $38,1 \%$ do N, 46,34\% do P, 40,19\% do S, 42,68\% do K, 13,19\% do Ca, 25,04\% do Mg, 40,63\% do Cu, 19,49\% do Zn e 17,73\% do B. Em cafeeiro cultivar Catuaí com quatro anos de idade, a alocação de macro e micronutrientes nos frutos, em relação à parte aérea, foi a seguinte: $27 \%$ do N, $27 \%$ do $\mathrm{P}, 23 \%$ do S, 35\% do K, $12 \%$ do Ca, $15 \%$ do Mg, $37 \%$ do $\mathrm{Cu}, 18 \%$ do $\mathrm{Zn}$ e $24 \%$ do B (Malavolta, 1986). Malavolta (1993) determinou a seguinte alocação percentual de nutrientes nos frutos para o cultivar Catuaí com 6,5 anos de idade: $25 \%$ do N, 32\% do P, $25 \%$ do S, $39 \%$ do K, menos de $10 \%$ do Ca, $14 \%$ do $\mathrm{Mg}$, menos de $10 \%$ do $\mathrm{Cu}$, menos de $10 \%$ do Zn e $21 \%$ do B. Depreendese, pelos valores apresentados, que a exportação de nutrientes pelo cafeeiro é considerável, ou seja, grande parte dos elementos contidos nos frutos deixa a propriedade. A exportação pode ser maior quando a palha, rica em nutrientes, não for devolvida ao cafezal. Além dos benefícios proporcionados por ser fonte de matéria orgânica, ela contém quantidade significativa de nutrientes, principalmente de K. Correa et al. (1983) observaram que a maior demanda de nutrientes por cafeeiro Catuaí, em anos de alta produtividade, ocorre em função da frutificação, enquanto nos anos de baixa produtividade a demanda é maior pela parte vegetativa.

\section{CONCLUSÕES}

A variedade Icatu Amarelo IAC-3282 foi a mais produtiva no ambiente com restrição de nutrientes, enquanto MG1192 e Catuaí Vermelho IAC-99 mostraram-se mais produtivas em ambientes com alto suprimento de nutrientes.

Não houve diferenças entre os cultivares na alocação relativa de nutrientes no caule.

A alocação de N, P, K e Mg no caule foi maior no nível baixo de adubação em comparação aos demais níveis.

Diferenças entre cultivares na alocação relativa de nutrientes em ramos só se verificaram no nível baixo de fertilização para P e Ca e no nível normal para N.

Considerando a média de alocação relativa de nutrientes nos frutos, para as quatro cultivares, no nível normal de adubação, verificou-se que eles possuem 38,1\% do N, 46,34\% do P, 40,19\% do S, 42,68\% do K, 13,19\% do Ca, 25,04\% do Mg, 40,63\% do Cu, 19,49\% do Zne 17,73\% do B.

\section{AGRADECIMENTOS}

Ao Consórcio Brasileiro de Pesquisa e Desenvolvimento do Café (CBP\&D-Café), pelo financiamento do projeto.

\section{REFERÊNCIAS}

Baligar VC \& Fageria NK (1998) Plant nutrient efficiency: towards the second paradigm. In: Siqueira, J.O. et al. (Eds.). Inter-relação fertilidade, biologia do solo e nutrição de plantas. Lavras, Sociedade Brasileira de Ciência do Solo. p.183 - 204.

Bingham FT (1982) Boron. In: Page AL (Ed.). Methods of soil analysis: chemical and microbiological properties. Madson, American Society of Agronomy. p.431 - 447.

Braga JM \& Defelipo BV (1974) Determinação espectrofotométrica de $\mathrm{P}$ em extratos de solo e material vegetal. Revista Ceres, 21:73-85.

Cataldo DA, Haroon M, Schrader, LE \& Younes V.L (1975) Rapid colorimetric determination of nitrate in plant tissue by nitration of salicilic acid. Communications in Soil Science and Plant Analysis, 6:71-80.

Correa JB, Garcia AWR \& Costa PC (1983) Extração de nutrientes pelos cafeeiros Mundo Novo e Catuaí. In: Congresso Brasileiro de Pesquisa Cafeeira, Poços de Caldas. Anais, IBC/GERCA. p.117-183.

Furlani AMC et al. (1984) Avaliação de genótipos de arroz quanto à eficiência na utilização de fósforo em solução nutritiva e em solo. Revista Brasileira de Ciência do Solo, 7:291-302.

Guimarães PTG, Garcia AWR, Alvarez VVH, Prezotti LC, Viana AS, Miguel AE, Malavolta E, Corrêa JB, Lopes AS, Nogueira, FD \& Monteiro AVC (1999) Cafeeiro. In: Ribeiro, AC, Guimarães PTG \& Alvarez V. VH (Eds.). Recomendações para o uso de corretivos e fertilizantes em Minas Gerais, 5a Aproximação. Viçosa, Comissão de Fertilidade do Solo do Estado de Minas Gerais - CFSEMG. p.289-302.

Jackson ML (1958) Soil chemical analysis. New Jersey, Prentice Hall. 498 p.

Johnson CM \& Ulrich A (1959) Analytical methods for use in plants analysis. Berkeley, California Experimental Station. p. 25-78. (Bull. 766).

Jones Junior JB, Wolf B \& Mills HA (1991) Plant analysis handbook: a practical sampling, preparation, analysis, and interpretation guide. Georgia, Micro-Macro Publishing. 213p.

Laviola BG, Martinez HEP, Souza RB \& AlvarezV VH (2007) Dinâmica de $\mathrm{P}$ e $\mathrm{S}$ em folhas, flores e frutos de cafeeiro arábico em três níveis de adubação. Bioscience Journal, 23:29-40.

Malavolta, E (1993) Nutrição mineral e adubação do cafeeiro. São Paulo, Editora Agronômica Ceres Ltda. 210p.

Malavolta, E (1986) Nutrição, adubação e calagem para o cafeeiro. In: Rena AB, Malavolta E, Rocha M, Yamada T.(Eds.) Cultura do cafeeiro: fatores que afetam a produtividade. Piracicaba, Associação Brasileira para Pesquisa da Potassa e do Fosfato. p.165-274.

Mantovani EC \& Costa LC (1998) Manual do SISDA 2.0. In: Workshop Internacional sobre Manejo Integrado das Culturas e Recursos Hídricos. Viçosa, Departamento de Engenharia Agrícola, 153p.

Morais EJ et al. (1990) Biomassa e eficiência nutricional de espécies de eucalipto em duas regiões bioclimáticas de Minas gerais. Revista Brasileira de Ciência do Solo, 14:353-362.

Moura WM, Pereira AA, Lima PC, Utida MK \& Castro NM (2000) Ensaio regional de linhagens comerciais de cultivares de café arábica. In: Simpósio de Pesquisas dos Cafés do Brasil. Anais, EMBRAPA. p.484.

Vose PB. (1987) Genetical aspects of mineral nutrition - progress to date. In: Gabelman HW \& Louhman (Eds.). Genetic aspects of plant mineral nutrition. Dordrecht, Boston \& Lancaster. p.3-13. 